\title{
Capítulo 15. Utilidad del mapeo tridimensional en pacientes con miocardiopatía arritmogénica (displasia arritmogénica del ventrículo derecho)
}

\author{
Chapter 15. Usefulness of three-dimensional mapping \\ in patients with arrhythmogenic cardiomyopathy \\ (arrhythmogenic right ventricular dysplasia)
}

\author{
Carlos A. Restrepo
}

Hospital Manuel Uribe Ángel, Envigado, Colombia

Recibido el 16 de noviembre de 2015; aceptado el 18 de enero de 2016

\section{Introducción}

Las cardiopatías arritmogénicas son enfermedades genéticamente determinadas, que producen daño progresivo del miocardio, tienen una alta probabilidad de causar muerte súbita por arritmias cardiacas y se manifiestan por lo general antes de los 35 años. La forma de presentación mejor conocida afecta principalmente al ventrículo derecho y se denomina displasia arritmogénica del ventrículo derecho, aunque en algunas oportunidades puede ocasionar también compromiso del ventrículo izquierdo. Debe sospecharse en todos los pacientes jóvenes con un corazón aparentemente normal que sufran síncope asociado a taquicardia ventricular o muerte súbita arrítmica ${ }^{1}$.

Es una enfermedad de carácter autosómico dominante con penetración variable y expresión incompleta. Se observa con mayor frecuencia en el sexo masculino y pueden encontrarse antecedentes familiares en aproximadamente un 30 a 50\% de los pacientes afectados. La edad al momento del diagnóstico es de 30 a 40 años. Su frecuencia varía ampliamente según las series y la región geográfica, pero se cree que representaría el $5 \%$ de las muertes súbitas en menores de 65 años y tendría una prevalencia que va de $1 / 2.000$ a $1 / 10.000^{1}$.

Se caracteriza por una sustitución progresiva del miocardio por tejido adiposo o fibroadiposo. Esta infiltración constituye un sustrato para la inestabilidad eléctrica y lleva a arritmias auriculares y ventriculares; sin embargo, es la tendencia a producir arritmias ventriculares malignas y muerte súbita lo que explica la gravedad de esta enfermedad ${ }^{1}$.

Una de sus variedades, denominada queratodermia palmoplantar (enfermedad de Naxos), ha permitido estudiar en detalle su fisiopatología. Este tipo de displasia arritmogénica es causado por una mutación en el gen de la placoglobina, cuyo producto es un componente de los desmosomas y las uniones celulares. En otros casos con presentación familiar se han identificado alteraciones de la desmoplaquina y de la placofilina 2. Se han identificado también otras mutaciones presuntamente causantes de esta enfermedad en la placoglobina, así como en otros genes desmosómicos de la desmocolina 2 y la desmogleína $2^{1}$. En la actualidad se considera

Correo electrónico: carlos24@une.net.co 
que la disfunción desmosómica es la vía final común en la patogenia de casi todos los casos de displasia arritmogénica. La integridad estructural y funcional del tejido cardiaco se basa en los desmosomas, las uniones tipo adherens (uniones adherentes) y las uniones tipo gap (uniones estrechas) situadas en los discos intercalares. La integridad de los desmosomas es necesaria para mantener el acoplamiento eléctrico y los mecanismos de señalización en la regulación del crecimiento, la diferenciación y el desarrollo celulares ${ }^{1}$. Sin embargo, algunos genes no desmosómicos se han asociado también con casos de displasia arritmogénica, como mutaciones en el gen del factor de crecimiento transformador B-3 (TGFB3), que modula la expresión de las proteínas de contacto celular y el gen del receptor de rianodina $2(R y R 2)^{1}$.

La mayoría de veces, el proceso de reemplazo fibroso afecta las áreas posterior e inferior del tracto de entrada del ventrículo derecho vecino a la válvula tricúspide, al infundíbulo anterior y al vértice cardiaco, formando lo que se conoce como triángulo de la displasia. La enfermedad puede causar anomalías de la contracción generales o regionales, alteraciones de la función sistólica y diastólica del ventrículo derecho, formación de aneurismas y dilatación e hipocinesia. El tabique interventricular suele estar preservado, por lo cual las biopsias endomiocárdicas que generalmente se obtienen del septum apical pueden no ser diagnósticas. La insuficiencia cardiaca sintomática puede ser una manifestación de la displasia arritmogénica y la mayoría de veces se produce en estadios avanzados de la enfermedad. Esto se debe a que la enfermedad es progresiva y termina por producir también compromiso del ventrículo izquierdo ${ }^{1}$.

El electrocardiograma puede ser anormal, observándose algunas veces ondas $T$ invertidas y ondas épsilon en las derivaciones V1-V3. Las ayudas diagnósticas de mayor utilidad son la ecocardiografía y la resonancia magnética, para las cuales se han establecido algunos criterios diagnósticos precisos ${ }^{1}$.

En los pacientes con displasia arritmogénica, el implante de desfibrilador mejora el pronóstico y la supervivencia si se aplica a una población de alto riesgo y como prevención secundaria. Sin embargo, los criterios para la selección óptima de pacientes para el implante de desfibrilador como prevención primaria de la muerte súbita, todavía no son claros en esta patología. Es de suponer que los estudios genéticos tendrán gran importancia en este aspecto en el futuro cercano, y permitirán detectar con prontitud los casos de alto riesgo arrítmico.

\section{Utilidad del mapeo tridimensional}

Los métodos recientes que permiten el mapeo tridimensional computarizado de las cavidades cardiacas, han sido de gran valor para el estudio de la fisiopatología de esta enfermedad y para su tratamiento. Se ha observado que las cicatrices del ventrículo derecho identificadas mediante mapas electroanatómicos, corresponden a áreas de depleción miocárdica y están correlacionadas con los signos histopatológicos de atrofia miocárdica y sustitución fibroadiposa.

Corrado et al. ${ }^{2}$ demostraron que los mapas de voltaje pueden ser útiles en el diagnóstico diferencial con otras arritmias y las formas tempranas de la displasia arritmogénica. De hecho, en los pacientes con arritmias ventriculares originadas en el tracto de salida derecho y con ecocardiografías normales, la presencia de un área de regiones de bajo voltaje identificó un subgrupo con alto riesgo de recurrencia de taquicardia ventricular e incluso de muerte súbita durante el seguimiento clínico ${ }^{3}$.

Wijnmaalen et al. ${ }^{4}$ informaron que los pacientes con taquicardia ventricular derecha asociada a la presencia de cicatrices, con o sin un diagnóstico de displasia establecido según los criterios del Task Force, tenían una tasa de recurrencia de taquicardia superior a la de los pacientes sin cicatrices electroanatómicas. Todos estos datos confirman que los mapas de voltaje podrían ser un instrumento diagnóstico importante con implicaciones pronósticas y terapéuticas.

Otros autores recomiendan realizar biopsias endomiocárdicas guiadas por mapa tridimensional, debido a que esta herramienta permite delimitar las áreas enfermas de las sanas $^{5,6}$.

La taquicardia ventricular en los casos de displasia arritmogénica, es el resultado de circuitos de reentrada relacionados con una cicatriz, de manera similar a lo que se observa en el contexto del paciente con infarto de miocardio. La ablación por catéter con mapas de voltaje del ventrículo derecho, mediante el empleo de técnicas de elaboración de mapas convencionales o electroanatómicos, puede aportar resultados favorables a corto plazo. Sin embargo, como consecuencia de la progresión de la enfermedad, las recurrencias de las arritmias a partir de nuevos sustratos arritmogénicos son frecuentes ${ }^{7}$.

En el plano endocárdico, las regiones con potenciales de bajo voltaje $(<1,5 \mathrm{mV})$, con larga duración y fraccionados se correlacionan con pérdida de miocitos y reemplazo fibroadiposo, y confirman circuitos críticos para la taquicardia ventricular $^{8}$. La distribución endocárdica de estas cicatrices se extiende desde la válvula tricúspide y/o pulmonar a la pared libre del ventrículo derecho, y aunque pueden encontrarse en los aspectos septales de las regiones perivalvulares, no incluyen comúnmente el ápex ${ }^{9}$. Los mapas tridimensionales del ventrículo izquierdo muestran que cuando hay compromiso se localiza frecuentemente en las regiones perivalvulares ${ }^{9}$.

El compromiso epicárdico es común en esta patología y en general es más extenso que el endocárdico ${ }^{10}$. En este sentido, se recomienda que el umbral de detección de cicatriz sea de $1 \mathrm{mV}$ para mejorar la distinción respecto a la grasa epicárdi$\mathrm{ca}^{11}$. Se ha sugerido que la determinación del voltaje unipolar endocárdico puede predecir cicatrices epicárdicas y el umbral de $<5,5 \mathrm{mV}$ se asocia con compromiso epicárdico ${ }^{12}$.

La técnica de ablación no está completamente establecida e incluye lesiones lineares que seccionan una cicatriz uniéndola a regiones eléctricamente inertes (anillos valvulares, otra cicatriz), lesiones dirigidas a potenciales tardíos con extensión de 2-3 cm sobre esta zona o a la reciente técnica de descanalización ${ }^{10,13}$. En esta última se hace un mapeo de alta densidad en ritmo sinusal encontrando zonas de despolarización retardada que se hallan en el borde de la cicatriz y pueden ser objetivo de la ablación (figs. 1 y 2$)^{14}$.

Muchos autores consideran que las indicaciones actuales para la ablación por catéter en pacientes con displasia arritmogénica son la presencia de taquicardia ventricular monomórfica bien tolerada con formas localizadas de la enfermedad, siempre que no mejoren con la medicación, además de los casos de taquicardia ventricular de presentación frecuente, incesante o que se relaciona con descargas múltiples por parte de un desfibrilador implantado. 


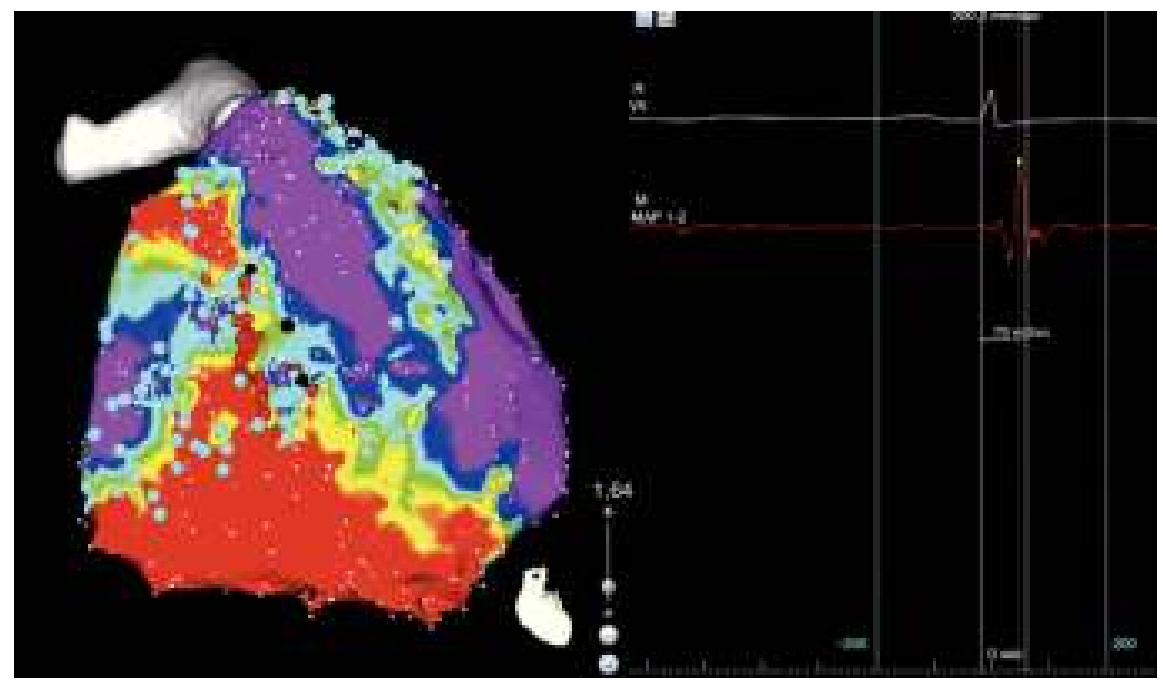

Figura 1 Se muestra una zona de bajo voltaje que corresponde a cicatriz (rojo), la zona que rodea la cicatriz o border (verde) y el tejido eléctricamente sano (violeta). Tomada con permiso de Díaz et al. ${ }^{14}$.

Kusano et al. ${ }^{15}$ reportan ablaciones exitosas mediante la aplicación de radiofrecuencia en istmos críticos. Por su parte, Nair et al. ${ }^{16}$ y Della Bella et al. ${ }^{17}$ realizaron ablaciones exitosas induciendo taquicardias inestables desde el punto de vista hemodinámico, que lograron ser mapeadas por técnica de no-contacto (Array, St. Jude Medical).

En términos generales, se podría afirmar que todas las técnicas de ablación usadas en la taquicardia ventricular relacionada con cardiopatía isquémica con infartos previos, son válidas para la ablación de la displasia arritmogénica, debido a las similitudes en su fisiopatología. En todo caso, estas técnicas requieren mapeo tridimensional para su realización ${ }^{9-11,13-22}$. Sin embargo, debido a la naturaleza progresiva de esta enfermedad, son comunes las recurrencias y la única terapia que puede aportar mejoría en la supervivencia de estos pacientes es el implante de un dispositivo desfibrilador.

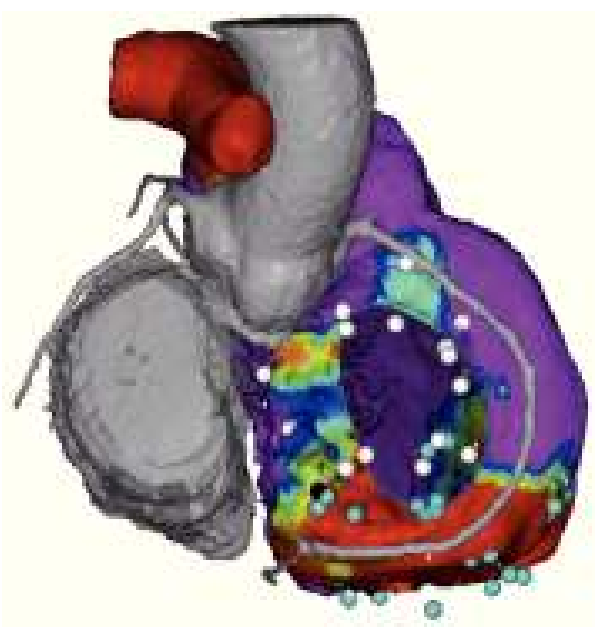

Figura 2 Imagen fusionada con tomografía computarizada en la que se muestra curso de otras estructuras cardiacas con la base del ventrículo derecho incluyendo las arterias coronarias. Tomada con permiso de Díaz et al. ${ }^{14}$.

\section{Recomendaciones}

\section{Clase I}

- Se recomienda el procedimiento de ablación de arritmias mediante mapeo tridimensional en pacientes con displasia arritmogénica que deban ser llevados a ablación por taquicardia ventricular (nivel de evidencia C).

\section{Bibliografía}

1. Marcus FI, McKenna WJ, Sherrill D, et al. Diagnosis of arrhythmogenic right ventricular cardiomyopathy/dysplasia: proposed modification of the task force criteria. Circulation. 2010;121:1533-41.

2. Corrado D, Basso C, Leoni L, et al. Three-dimensional electroanatomic voltage mapping increases accuracy of diagnosing arrhythmogenic right ventricular cardiomyopathy/dysplasia. Circulation. 2005;14;111:3042-50.

3. Corrado D, Basso C, Leoni L, Tokajuk B, Turrini P, Bauce B, et al. Three-dimensional electroanatomical voltage mapping and histologic evaluation of myocardial substrate in right ventricular outflow tract tachycardia. J Am Coll Cardiol. 2008;51:7314.

4. Wijnmaalen AP, Schalij MJ, Bootsma M, et al. Patients with scar-related right ventricular tachycardia: determinants of long-term outcome. J Cardiovasc Electrophysiol. 2009;20: 1119-27.

5. Pieroni M, Dello Russo A, Marzo F, et al. High prevalence of myocarditis mimicking arrhythmogenic right ventricular cardiomyopathy: differential diagnosis by electroanatomic mapping-guided biopsy. J Am Coll Cardiol. 2009;53:6.

6. Avella A, D'Amati G, Pappalardo A, et al. Diagnostic value of endomyocardial biopsy guided by electroanatomic voltage mapping in arrhythmogenic right ventricular cardiomyopathy/dysplasia. J Cardiovasc Electrophysiol. 2008;19:112734.

7. Verma A, Kilicaslan F, Schweikert RA, et al. Short- and longterm success of substrate-based mapping and ablation of ventricular tachycardia in arrhythmogenic right ventricular dysplasia. Circulation. 2005;111:3209-16. 
8. Kiès P, Bootsma M, Bax J, Schalij MJ, van der Wall EE. Arrhythmogenicright ventricular dysplasia/cardiomyopathy: screening, diagnosis, and treatment. Heart Rhythm. 2006;3:225-34.

9. Marchlinski FE, Zado E, Dixit S, et al. Electroanatomic substrate and outcome of catheter ablative therapy for ventricular tachycardia in the setting of right ventricular cardiomyopathy. Circulation. 2004;110:2293-8.

10. Garcia FC, Bazan V, Zado ES, Ren JF, Marchlinski FE. Epicardial substrate and outcome with epicardial ablation of ventricular tachycardia in the arrhythmogenic right ventricular cardiomyopathy/dysplasia. Circulation. 2009;120:366-75.

11. Cano $O$, Hutchinson $M$, Lin $D$, et al. Electroanatomic substrate and ablation outcome for suspected epicardial ventricular tachycardia in left ventricular nonischemic cardiomyopathy. J Am Coll Cardiol. 2009;54:799-808.

12. Polin GM, Haqqani H, Tzou W, et al. Endocardial unipolar voltage apping to identify epicardial substrate in arrhythmogenic right ventricular cardiomyopathy/dysplasia. Heart Rhythm. 2011;8:76-83.

13. Berruezo A, Fernández-Armenta J, Mont L, et al. Combined endocardial and epicardial catheter ablation in arrhythmogenic right ventricular dysplasia incorporating scar dechanneling technique. Circ Arrhythm Electrophysiol. 2012;5:111-21.

14. Diaz A, Acosta J, Penela D, et al. Ablación de taquicardia ventricular en displasia arritmogénica del ventrículo derecho. Rev Colom Cardiol. 2015;22:88-96.

15. Kusano KF, Emori T, Morita $\mathrm{H}$, et al. Ablation of ventricular tachycardia by isolating the critical site in a patient with arrhythmogenic right ventricular cardiomyopathy. J Cardiovasc Electrophysiol. 2000;11:102-5.
16. Nair M, Yaduvanshi A, Kataria V, Kumar M. Radiofrequency catheter ablation of ventricular tachycardia in arrhythmogenic right ventricular dysplasia/cardiomyopathy using non-contact electroanatomical mapping: single-center experience with follow-up up to median of 30 months. J Interv Card Electrophysiol. 2011;31:141-7.

17. Della Bella P, Pappalardo A, Riva S, et al. Non-contact mapping to guide catheter ablation of untolerated ventricular tachycardia. Eur Heart J. 2002;23:742-52.

18. Reithmann C, Hahnefeld A, Remp T, et al. Electroanatomic mapping of endocardial right ventricular activation as a guide for catheter ablation in patients with arrhythmogenic right ventricular dysplasia. Pacing Clin Electrophysiol. 2003;26:1308-16.

19. Dalal $D$, Jain $R$, Tandri $H$, et al. Long-term efficacy of catheter ablation of ventricular tachycardia in patients with arrhythmogenic right ventricular dysplasia/cardiomyopathy. J Am Coll Cardiol. 2007;50:432-40.

20. Arruda M, Armaganijan L, Fahmy T, Di Biase L, Patel D, Natale A. Catheter ablation of ventricular tachycardia in arrhythmogenic right ventricular dysplasia. J Interv Card Electrophysiol. 2009;25:129-13.

21. Bai R, Di Biase L, Shivkumar K, et al. Ablation of ventricular arrhythmias in arrhythmogenic right ventricular dysplasia/cardiomyopathy: arrhythmia-free survival after endo-epicardial substrate based mapping and ablation. Circ Arrhythm Electrophysiol. 2011;4:478-85.

22. Philips B, Madhavan S, James C, et al. Outcomes of catheter ablation of ventricular tachycardia in arrhythmogenic right ventricular dysplasia/cardiomyopathy. Circ Arrhythm Electrophysiol. 2012;5:499. 